\title{
EDITORIAL
}

\section{Caring for the orphan's orphan: treatment of patients with portopulmonary hypertension}

\author{
S.M. Kawut
}

$\mathbf{P}$ atients with portopulmonary hypertension (PPHTN) are incredibly unfortunate. By definition, these patients have portal hypertension, leading to well-known lifethreatening gastrointestinal, neurological, infectious, haematological and renal complications requiring liver transplantation for cure.

If this were not enough, these patients also have pulmonary arterial hypertension (PAH). The coexistence of portal hypertension and $\mathrm{PAH}$ frequently makes patients with this rare (or "orphan") condition medical "untouchables". PPHTN patients with progressive hepatic dysfunction and other complications are often deemed to be too "high-risk" for placement of a transjugular intrahepatic portosystemic shunt or liver transplantation, making them destined for a poor outcome. Even those who may be acceptable candidates for liver transplantation commonly face a complicated perioperative course.

The situation is not much better from the vantage of their lung disease, despite these patients having the third most common form of $\mathrm{PAH}$ in a recent population-based epidemiological study from France [1]. While there is some evidence regarding medications which should be avoided in PPHTN [2], therapeutic approaches used to treat other forms of PAH have never been trialled in PPHTN, are potentially complicated by coexistent liver disease, and are used in PPHTN without the evidence base which now exists for other types of PAH.

Many individuals with PPHTN have a history of (or are currently) using alcohol or illicit drugs, addictions commonly linked to the aetiology of their liver disease, and some have HIV infection, all accompanied by significant social stigma. These comorbidities often further limit access to specialised centres and available treatments. The sum of these burdens and the high toll of the disease have proved significant obstacles to successful advocacy on behalf of this patient group. Who can blame industry sponsors, scientific advisory committees and regulatory boards for excluding these patients from clinical trials of new therapies for PAH?

It is against this backdrop that we should consider the multicentre cohort of consecutively treated patients with

STATEMENT OF INTEREST: None declared.

CORRESPONDENCE: S.M. Kawut, Division of Pulmonary, Allergy, and Critical Care, Columbia University College of Physicians and Surgeons, 622 W. 168th Street, PH 8E, Rm. 101, New York City, NY, 10032, USA. Fax: 1 2123422201. E-mail: sk2097@columbia.edu
PPHTN published by HoEPER et al. [3] in the current issue of the European Respiratory Journal. These investigators retrospectively studied 31 patients who started treatment with either oral bosentan, an endothelin (ET)-receptor antagonist, or inhaled iloprost, a prostacyclin analogue, in a nonrandomised fashion between 1999 and 2004. The investigators included all patients with PPHTN evaluated at Hannover Medical School, Hannover, University of Leipzig, Leipzig and Carl-GustavCarus University, Dresden (all Germany) during the study period, except for two who did not receive specific medical therapy. The physicians prescribed iloprost and bosentan using standard dosing regimens and based the therapeutic choice on their (and the patients') preferences. The primary outcome of interest in this study was survival; however multiple secondary outcomes were assessed.

Patients in the two groups were quite similar in terms of baseline haemodynamics, functional class and distance walked in 6 mins. Patients who were treated with bosentan were somewhat more likely to have alcoholic cirrhosis and were less likely to have autoimmune hepatitis than patients treated with iloprost. Also, patients treated with bosentan may have had less severe hepatic dysfunction compared with those who were treated with iloprost, judging from the somewhat greater percentage of Child-Pugh class A patients and somewhat lower mean Model for End Stage Liver Disease (MELD) score in the former group compared with the latter. As bosentan may cause elevated liver transaminases in a minority of patients, these imbalances possibly reflected physician (and patient) preference to use this medication in individuals with less severe liver disease and iloprost in those with more severe disease.

Patients who were treated with bosentan had 1- and 3-yr survival rates of 94 and $89 \%$, respectively, significantly higher than those of patients treated with iloprost, which were 77 and $46 \%$, respectively. Interestingly, the survival of patients treated with bosentan exceeded those of patients in recent cohort studies from other centres [4,5]. Multivariate analyses, which appropriately adjusted for potential confounders including distance walked in 6 mins, right atrial pressure and MELD score confirmed the finding of a higher risk of death in patients treated with iloprost compared with the risk of those treated with bosentan, making it less likely that baseline differences between the groups in these variables accounted for the findings.

Further analyses of changes in haemodynamics over time appear to support the survival results, but were limited by 
varying times of assessment and only a subset of patients being reassessed after drug initiation. The change in distance walked over time was not different between the two groups. Of the 13 patients who received inhaled iloprost, only three continued this therapy for the 3-yr study period without clinical worsening. Of the 18 patients treated with bosentan, 14 continued treatment without clinical events. Side-effects appeared to be minimal with either therapy, and only one patient receiving bosentan had elevation in transaminases, which normalised after dose reduction.

Several statements may be made from these data. First, this is one of the larger studies of the impact of therapy in PPHTN in the recent era [4-8]. While this distinction is more a testament to the relatively small number of patients with PPHTN included in epidemiological studies rather than the absolute magnitude of the current study sample, clinical researchers need to follow the lead of HOEPER et al. [3] in forming multicentre national and international consortia in order to study meaningful numbers of these patients over time. The National Institutes of Health have supported such an effort in the USA.

Secondly, patients initially treated with bosentan clearly had better survival compared with patients initially treated with iloprost. This finding could be explained in several ways. Underlying differences in the patients and in the allocation of medical therapy could account for this finding. The complexity of treatment decisions inevitably results in incomparability between groups of patients prescribed different therapies, which can not be fully resolved by statistical analysis because these decisions can not be adequately characterised by a few measured variables $[9,10]$. Even with appropriate multivariate analysis, confounders which are imprecisely measured or unmeasured could still result in the appearance of a significant effect of therapy on outcomes where there is really none. Furthermore, differences in compliance with therapy between the groups could also potentially lead to disparities in outcome, even if the therapies actually have similar efficacy when used under ideal conditions. Greater difficulty in using inhaled iloprost as prescribed compared with bosentan could thus have led to the findings. Finally, it is indeed possible that oral bosentan may actually be more efficacious and/or effective than inhaled iloprost in patients with PPHTN.

Unfortunately, it is impossible to know for certain which of these conclusions actually holds true in an observational study. Randomised controlled trials (RCTs) that contradict promising epidemiological studies of therapy remind us of the restrained enthusiasm we should have about results from nonrandomised studies. Therefore, these findings should spur the development of RCTs either focused on PPHTN or (at the least) including patients with this disease.

Thirdly, if bosentan is more effective than iloprost, this supports the importance of ET-1 in the progression of PPHTN. One study has shown that patients with PPHTN have higher ET-1 levels compared with patients with portal hypertension without this complication [11]. Differences in ET1 production or sensitivity to its effects could explain why only approximately one out of 20 patients with portal hypertension develops PPHTN.
Fourthly, the current study implies that both bosentan and iloprost may be utilised in patients with PPHTN and ChildPugh class A liver disease without significant side-effects, further supported by the recent publication of a European registry of bosentan use [12]. Of course, the former study is relatively small, and the latter subject to voluntary reporting, so that the actual safety of this approach is still unknown, as emphasised by the authors. Long-term epidemiological studies of consecutive patients with standardised follow-up and RCTs would be the best way to establish the safety of the respective treatment approaches.

Finally, this manuscript frames the clinical equipoise which surrounds the medical treatment and liver transplantation of patients with portopulmonary hypertension. The experience of HOEPER et al. [3] suggests that bosentan may be preferable; however, there is no firm evidence that one particular therapeutic approach is superior (in terms of functional status, quality of life, outcomes or safety) to another or to placebo. This is precisely the setting in which randomised controlled trials are not only acceptable, but also ethically imperative in order to prevent the use of expensive and inconvenient therapies that may be ineffective and possibly risky in patients. Protests that the disease is too uncommon or complicated for randomised controlled trials are reminiscent of those heard not so long ago in reference to the orphan disease of idiopathic pulmonary arterial hypertension, a cause now adopted by federal and industry sponsorship with a proven treatment pedigree. The work of HOEPER et al. [3] is a mandate to establish a therapeutic bloodline by which to treat our patients with portopulmonary hypertension, so they may also benefit from the recent advances which have improved outcomes in other forms of pulmonary arterial hypertension.

\section{REFERENCES}

1 Humbert M, Sitbon O, Chaouat A, et al. Pulmonary arterial hypertension in France: Results from a national registry. Am J Respir Crit Care Med 2006; 173: 1023-1030.

2 Provencher S, Herve $P$, Jais $X$, et al. Deleterious effects of $\beta$ blockers on exercise capacity and hemodynamics in patients with portopulmonary hypertension. Gastroenterology 2006; 130: $120-126$.

3 Hoeper MM, Seyfarth HJ, Hoeffken G, et al. Experience with inhaled iloprost and bosentan in portopulmonary hypertension. Eur Respir J 2007; 30: 1096-1102.

4 Herve P, Le Pavec J, Sztrymf B, Decante B, Savale L, Sitbon O. Pulmonary vascular abnormalities in cirrhosis. Best Pract Res Clin Gastroenterol 2007; 21: 141-159.

5 Kawut SM, Taichman DB, Ahya VN, et al. Hemodynamics and survival of patients with portopulmonary hypertension. Liver Transpl 2005; 11: 1107-1111.

6 Fix OK, Bass NM, De Marco T, Merriman RB. Long-term follow-up of portopulmonary hypertension: effect of treatment with epoprostenol. Liver Transpl 2007; 13: 875-885.

7 Sussman N, Kaza V, Barshes N, et al. Successful liver transplantation following medical management of portopulmonary hypertension: a single-center series. Am J Transplant 2006; 6: 2177-2182.

8 Krowka MJ, Frantz RP, McGoon MD, Severson C, Plevak DJ, Wiesner RH. Improvement in pulmonary hemodynamics 
during intravenous epoprostenol (prostacyclin): a study of 15 patients with moderate to severe portopulmonary hypertension. Hepatology 1999; 30: 641-648.

9 Vandenbroucke JP. Analytic approaches to observational studies with treatment selection bias. JAMA 2007; 297: 2077-2078.

10 Miettinen OS. The need for randomization in the study of intended effects. Stat Med 1983; 2: 267-271.
11 Benjaminov FS, Prentice M, Sniderman KW, Siu S, Liu P, Wong F. Portopulmonary hypertension in decompensated cirrhosis with refractory ascites. Gut 2003; 52: 13551362.

12 Humbert M, Segal ES, Kiely DG, Carlsen J, Schwierin B, Hoeper MM. Results of European post-marketing surveillance of bosentan in pulmonary hypertension. Eur Respir $J$ 2007; 30: 338-344. 